\title{
ERRATUM
}

Damien O. Joly · François Messier

\section{The distribution of Echinococcus granulosus in moose: evidence for parasite-induced vulnerability to predation by wolves?}

Published online: 9 December 2004

(C) Springer-Verlag 2004

\section{Oecologia (2004) 140:586-590}

Due to an unfortunate error Table 1 appeared incomplete. The complete table is printed below.

Table 1 Characteristics of the three adjacent study areas (L, M and $\mathrm{H})$ in southwestern Québec where moose lungs were collected for this study. Moose population data are from Messier et al. (1989). Green's coefficient of dispersion is denoted by $C$. Standard errors are indicated in parentheses

\begin{tabular}{llll}
\hline \multicolumn{4}{l}{ Study area } \\
\cline { 2 - 4 } & $\mathrm{L}$ & $\mathrm{M}$ & $\mathrm{H}$ \\
\hline Moose population characteristics & & & \\
Moose density (moose $\left./ \mathrm{km}^{2}\right)$ & 0.17 & 0.23 & 0.37 \\
Hunting rate $(\% /$ year) & $20-25$ & $20-25$ & $3-4$ \\
Wolf density (wolves/100 $\mathrm{km}^{2}$ ) & 0.36 & 0.82 & 1.38 \\
Predation rate (\%/year) & 6.1 & 10.7 & 19.3 \\
Echinococcus granulosus population characteristics & \\
Number of moose examined & 37 & 45 & 131 \\
Prevalence (\%) & 32 & 29 & 59 \\
Mean intensity & 7.2 & 13.3 & 28.3 \\
C & 0.205 & 0.116 & 0.044 \\
& $(0.107)$ & $(0.049)$ & $(0.05)^{\mathrm{a}}$ \\
\hline
\end{tabular}

${ }^{\mathrm{a}}$ Corrected for sample size

The online version of the original article can be found at http:// dx.doi.org/10.1007/s00442-004-1633-0

D. O. Joly $(\bowtie) \cdot$ F. Messier

Department of Biology,

University of Saskatchewan,

112 Science Place, Saskatoon, SK, Canada, S7N 5E2

E-mail: do_joly@yahoo.ca

Tel.: + 1-718-220-5892

Fax: + 1-718-220-0741

Present address: D. O. Joly

Field Veterinary Program, Wildlife Conservation Society, 2300 Southern Boulevard, Bronx, NY 10460, USA 